occurred to me that itwould be very instructive to endeavour to explain the possible cause of the abnormal temperature. The usual explanation of elevation of temperature is, to put it shortly, increased tissue metabolism, and the cause of low temperature exactly the reverse. In this case, how ever, it appears to me that this explanation does nothold good, as the condition of the patient-viz., that of exaggerated restlessness and delirium--was the same during the period of low as during the period of high temperature, at any rate as far as the elevation to $104^{\circ}$. The possible explanation appeared to be that the so-called "heat-regulating" centre in the cortex had been directly interfesed with by the disease of its membranes, and this case seems at least to suggest some corroboration of the presence of this cortical centre. That the thickened, gelatinous membranes must have affected the functions of the subjacent cortex cannot, I think, be doubted; and the fact of the facial paralysis and the evanescent limb paralysis a common feature in general paralysis of the insane, in which the naked-eye appearances of the membranes are exactly as in this case-I think corroborates this.

For permission to publish this case I am indebted to Dr. Seward, medical superintendent of the male department. Colney Hatch.

\section{PERFORATION DURING ENTERIC FEVER; RELAPSE ; RECOVERY.}

\section{BY RICHARD LAKE, L.R.C.P. LOND., \&e.}

ON March 28th last I was called in to see a young lady, aged nineteen, suffering from sickness and diarrhoea. She gave the following account of herself. Twenty-five days previously she was seized with romiting and diarrhoea during the night; the latter continued until four days before the above date, when she complained of more malaise than hitherto, and was given a saline purge. All her previous troubles now became exaggerated, and troublesome vomiting set in.

On examination her abdomen was slightly distended and tumid. Pain was present in the right iliac fossa, also gurgling. No spots were found. The vonit was clear and of a bright-green colour. The stools were of a vellow peasoupr consistence, with some floating flakes of mucus; fifteen stools during the last twenty-four hours. Temperature $103^{\circ}$; pulse 100 . Tongue red in centre; edges covered with a thick white fur; dry, but not cracked.

March 29th.-Bowels acted thirteen times during the day. Less vomiting. Liver duIness increased, with marked tenderness over the whole organ. Ice given to suck.

30th.-No vomiting until 5 P.M. One-third of a grain of morphia was given thrice in six hours to check the diarrhœea.

The temperature from the 28 th for five days fluctuated between $103^{\circ}$ and $101^{\circ} \mathrm{F}$., being only slightly influenced by antifebrin. The symptoms scarcely varied at all, excepting that the diarrhoea was checked by the three doses of morphia. During the next five days defervescence took place, the morning fall approaching or reaching normal, and the evening rise only once reaching $101^{\circ}$. During the four following days the chart was about this level, the temperature rising once to $1025^{\circ}$; this was due to constipation, as the temperature fell inmediately the bowels acted. (See chart.) On the night of April 9th pain was complained of in the abdomen, but was not severe, and was treated as colic by hot fomentations. As the pain continued and became more severe, the temperature rising, the patient at the same time getting an anxious expression, the abdomen was subjected to another examination, with the result of the discovery of a tender spot midway between the eighth right costal cartilage and the umbilicus. The hot fomentations were reapplied and nourishment given, chiefly brandy and raw beef juice. At mid-day on the 10th, the fall of temperature was so sudden, and symptoms of collapse so rapidly followed, the face becoming blanched, the tongue dry, and the pulse frequent and small, that perforation of the intestine was diagnosed, which belief was strengthened by the persistence of tenderness in the right hypochondrium, together with some ill-defined dulness there, with sharp shooting pain in the abdomen. Hot bottles were used to pack the patient in, and the following measures taken to endeavour to prolong life. The patient was kept in the dorsal decubitus, with Hexed legs and thighs, together with the application of hot fomentations to the abdomen. Of this period a chart is given, continued until all signs of inflammatory action had ceased-i.e., on April 14th. An ounce of brandy, with an ounce of raw beef juice, were given in the form of an enema every hour; also five minims of tincture of opium every hour by the mouth for the time the patient remsined awake, and then every two hours until the l2th; subsequently every four hours until the 14th, when it was discontinued entirely, and food again administered by the mouth. With regard to the general and local symptoms after the morning of the $11 \mathrm{th}$, green vomiting was again present, but was checked by sucking ice. The dulness in the right hypochondrium extended until it occupied an area of about four inches in diameter, forming a fairly well-defined

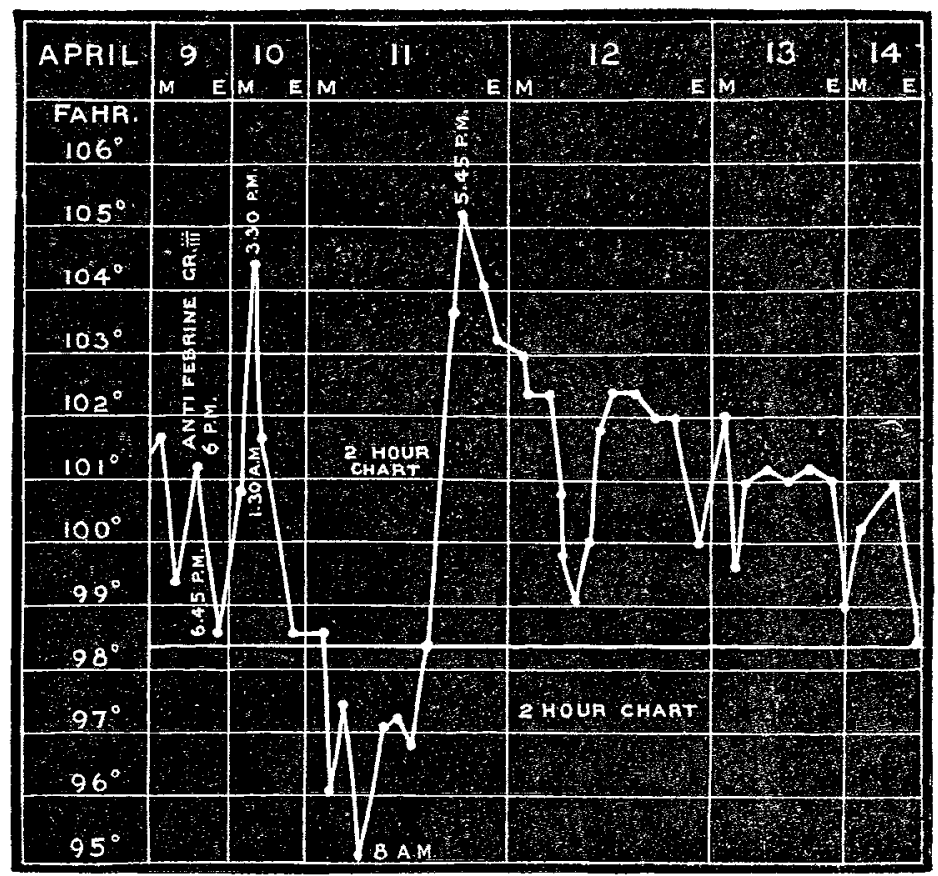

tumour, very painful on palpation ; this began to contract on the 14th, was almost imperceptible on the $28 \mathrm{th}$, and was entirely absent by the 10th of May. The pain was at all times the same, sharp and lancinating, and often accompanied by vomiting. The bowels were only moved by glycerine enemata, and there was much chalky matter in the motions. The breath was exceedingly offensive, and had a strong frecal odour. From the 14th to the 18th the temperature remained subnormal, with evening rises above the normal. On the latter day a relapse commenced, the temperature reaching $102 \cdot 6^{\circ}$ on the evening of the $22 \mathrm{nd}$, falling to the normal by the 27 th. There was one rise on the 26th up to $104 \cdot 2^{\circ}$, with voniting; this was explained by the bowels not having acted, as on the $7 \mathrm{th}$. The patient has lately returned to Scotland, and is quite well to all appearances.

Remarlis.-Any doubt remaining as to this case being enteric ferer is remover by the following facts. 1. The patient contracted the illness in a district where enteric was prevalent. 2. The main and house drains were very defective, the former blocked, as I learn from the medical officer of health for the town. 3. Several other pupils from the same house had the disease; the date of invasion being in all cases the same, and three deaths occurring, one of those first mentioned being under my care suffering from enteric. 4: Nurse Scarman, to whose skilful care the successful issue of the case was largely due, contracted enteric, and was treated at the Victoria Nursing Home, from which institution she came. The chief interest of the case lies in the period expressed on the chart-i. e., from April 9th to the 14th. It is obvious that some severe lesion had occurred on the evening of the 9th, as is shown by the sudden collapse and rapid rise of temperature which followed. Ur. Donald Hood has recorded several cases of venous thrombosis in the course of enteric fever, which have suggested perforation by their course and symptoms; but though collapse may be present in these cases, neither is the fall so low as $95^{\circ}$, nor is the rise afterwards so high. It is known that perforation may occur in a portion of the intestine bound down by Q 2 
lymph, the result of a localised peritonitis from the spread of inflammatory action from the ulcerated Peyer's patch, thus localising the mischief; and these are apparently the changes which took place here.

Barnes.

\section{Clinical atotes:}

\section{MEDICAL， SURGICAL，OBSTETRICAL，AND THERAPEUTICAL.}

\section{CASE OF DEFICIENT ESOPHAGUS.}

\section{By Charles Steele, M.D., F.R.C.S.}

THE following case appears to me to be of interest from both a surgical and an anatomical point of view.

I was lately asked to see in consultation an infant twentyfour hours old, who, shortly after being given nourishment, a little of which was taken readily, became very livid, had difficulty in breathing, and then returned the food and appeared no worse. The gentleman in attendance wisely introduced a sound, and found that it passed about five inches and encountered an impassable obstruction. $\mathrm{He}$ then asked me to see the child, and I repeated the sounding with the same conclusion. We diagnosed that there was either a membrane across the oesophagus, or that it ended in blind terminations; and I advised that through the night enemata of dessertspoonfuls of peptonised milk should be given every two hours, and that by daylight the stomach should be opened and the osophagus explored; if a mem brane could be made out across a continuous canal, that it should be perforated in order to give a hope of life; and that, if we found any distance existed between the extremities, we could do no more; the parents, however, might feel that every possible endeavour had been made to save their child's life. 'This was agreed upon, and the father willingly acceded. On the following afternoon I was asked to perform the operation. The infant took chloroform well. I opened the abdomen above the umbilicus in the middle line, exposed the stomach, and stitched it at four points to the skin, having some difficulty to keep the liver from protruding. The stomach was then opened, which was perfectly healthy, and of course empty. A bouoie was passed down the oesophagus as before, and another upwards from the stomach for a short distance; but they did not approach each other by what we judged to be an inch and a half. I then cut a gum-elastic catheter in half, and passed it from below, introduced up it a long slender steel probe, and pressed it upwards as much as was justifiable, in case the lower part of the tube might be twisted or narrowed, and capable of being rendered pervious. All was of no avail, however; so the stomach wound was closed with sutures, also the abdominal wound, and we felt sure that the cosophagus was deficient for about an inch and a half. The infant slept for some time, and died twenty-four hours afterwards. The next afternoon we made an examination, and found that the osophagus terminated above and below in blind rounded ends an inch and a half apart, and there was no cord or connexion between the parts. All the wounded portions were quite healthy, and the appearances led to the conclusion that had there been only a membranous occlusion a happy result might well have been hoped for.

\section{SUPPURATING HYDATID CYST OF THE LIVER PERFORATING THE LUNG; RECOVERY.}

By Fr.jycis W. JoshU 1 ,

SLRGEOX TO THE GREAT MLLYERN DISPENSARY.

Miss A. S—, aged fifty, of full habit, had in 1886 and 1887 several attacks of congestion of the liver and jaundice, but throughout December of the latter year felt perfectly well. On Jan. 6th, 1888, I was sent for, and found her in bed complaining of pain in the right shoulder and just below the right breast. Her countenance was anxious; temperature $101^{\circ}$; pulse 100 ; respiration hurried; no jaundice; dorsal decubitus. The liver was two inches below the ribs (the percussion note dull as high as the fifth interspace) ; its contour was smooth and regular; no fluctuation conld be detected. Gall-bladder distended ; no tenderness on palpation. Three weeks later the liver was three inches below the ribs, somewhat tender to the touch, and quite firm. The temperature during this period was generally normal during the say, rising to $100^{\circ}$ and $101^{\circ}$ in the evening. The bowels were constipated, and the evacuations untinged by bile. On Jan. 30th an incessant dry hacking cough set in, lasting ten days, which was relieved occasionally by morphia. The patient was now very ill and weak; there had been no rigors. On Feb. 9th she coughed up a pint and a half of pure pus. The following day a few hydatid cysts of small size appeared in the sputa. She complained of great sense of oppression and severe pain in the upper part of the chest, more especially on the left sicle. The lung and heart sounds were normal, and the chest was resonant throughout, save in the hepatic area, where there was absolute dulness and considerable bulging of the parietes. During the next few days hydatids in varying number were spat up daily, most of them larger in circumference than a crown piece; their emission was always accornpanied by most distressing paroxysms of coughing and extreme dyspnoa. On Feb. 17th the patient was very hoarse. At 1.30 P.M. she took a little solid food. At 2.30 P. M. sudden rush occurred from the mouth and nares of a thin fluid, hydatids, and blood-stained pus. I saw her a fer minutes later, when she was apparently moribund, no pulse at wrists, gasping inspirations (four or five in the minnte), extremities cold, face cyanosed, and completely unconscious. Two chamber utensils were shown me nearly full of blood-stained hydatids. I injected thirty minims of ether hypodermically, repeating the injections in a few minutes, with but slight result. Then, on the supposition that hæmorrhage had taken place into the cavity, I injected four grains of ergotine. The pulse returned gradually, but the cheeks and lips remained perfectly blancherl. I therefore injected into the rectum an ounce of brandy and half an ounce of turpentine with yelk of egg. Consciousness returned, and she was shortly able to swllow a small quantity of brandy-and-milk. On each succeeding day a few hydatids and some sanious matter were coughed up, until March 2nd, when the cough became more violent, and she expectorated a pint of pus during the day.On the 7 th she was jaundiced, and the temperature rose to $101^{\circ}$. On the 13th bile-stained hydatids were coughed up, and on the 14th a pint and a half of pure bile, which came up in varying quantities for a week, with one interval of two days. Then began an uninterrupted recovery, and on April 3rd she was able to bear renoval to another room. At this time the patient was greatly emaciated. The hypodermic punctures had sloughed, and a bulla four inches in diameter over the left tibia left a corresponding ulcer. The liver gradually receded to the lower margin of the ribs.

Remarlss.-The exceeding rarity of complete recovery in such cases renders the present one worthy, I think, of record. There was considerable difficulty in arriving at a correct ea:ly diagnosis, the (comparatively speaking) apparent smallness of the visceral enlargement, its firm and even outline, and absence of fluctuation leading one rather to the supposition that the case was one of malignant or amyloid disease.

Great Malvern.

\section{ON THE SMOKING OF STRAMONILM LEAVES, SIMULATING INSANITY.}

BY M. J. T. J. BLancard, SURg. M.A.

ON Aug. 30th, 1887, while on duty with a flying column at Leygi, Upper Burmah, I was called to see a man found unconscious near a well close to the outpost. The man was taken to hospital, and on examination the following srmptoms were observed. Insensibility, with complete relaxation of voluntary and involuntary muscles. Pulse small and compressible. Surface of body cold and clammy. Pupils not reacting to light, but fixed in a dilated position. Deglutition normal. Sphincters relaxed; motions and urine passed. Reflexes absent. Breathing at first stertorous, but gradually becoming quicker, with intervals of sighing. When spoken to in a loud voice the man answered with a moan. Evelids closed-not naturally, but as if done purposely. Head cool. 